\title{
Alcohol, Aspirin, and Gastrointestinal Bleeding
}

\author{
KERRY GOULSTON,* M.D., M.R.A.C.P. ; ALLAN R. COOKE, $†$ M.B., M.R.A.C.P.
}

\begin{abstract}
Cummary: In 20 healthy male subjects faecal blood - loss was measured by means of a chromium-51labelled red blood cell technique. Mean daily faecal blood loss associated with unbuffered aspirin ingestion was significantly increased by alcohol in the 13 subjects studied. In seven others alcohol alone did not cause gastrointestinal bleeding. These findings suggest that alcohol may accentuate gastrointestinal blood loss associated with unbuffered aspirin ingestion.
\end{abstract}

\section{Introduction}

It is a general clinical impression that aspirin and alcohol may be associated with erosive gastritis. Whereas there have been many investigations of the effect of aspirin on gastrointestinal blood loss and it is well established that unbuffered aspirin increases such loss (Smith and Smith, 1966), there have been no published studies of the effect of alcohol (ethanol) on gastrointestinal loss of blood. Furthermore, there have been no studies of gastrointestinal blood loss when alcohol and aspirin were taken together.

In the present investigation gastrointestinal blood loss was measured after the ingestion of aspirin alone, aspirin plus alcohol, and alcohol alone. It was found that alcohol did not itself cause gastrointestinal blood loss, but it did augment aspirin-induced gastrointestinal blood loss.

\section{Methods}

Studies of faecal blood loss from the ingestion of aspirin alone and aspirin plus alcohol were made in 13 healthy male volunteers. In seven other subjects alcohol alone was ingested. The ages of the 20 subjects ranged from 20 to 25 years and none had a history of gastrointestinal disease. Gastrointestinal blood loss was estimated by the wellestablished technique of labelling the red blood cells of each subject with sodium chromate-51 and counting radioactivity in the faeces (Goulston and Skyring, 1954). Collection of faeces was started 48 hours after labelling, a disposable paint tin being used for each 24-hour faecal specimen. Faeces were collected for 20 consecutive days. On the $6 \mathrm{th}, 7 \mathrm{th}$, and 8 th days, after a six-hour fast, seven tablets of soluble unbuffered aspirin (Disprin) were taken by each subject as follows: one tablet (300 mg. of aspirin in each tablet) at 6 p.m., one at 6.30 p.m., one at 7 p.m., two at 10 p.m., and two at 7 a.m. the following day. On the 14th, 15th, and 16th days seven tablets of aspirin were takcn in the same fashion and alcohol was also administered. Alcohol, in the form of undiluted Australian whisky $(31.8 \% \mathrm{w} / \mathrm{v}$ ethanol), was taken as follows: $60 \mathrm{ml}$. at 6 p.m., $60 \mathrm{ml}$. at 6.30 p.m., and $60 \mathrm{ml}$. at 7 p.m. a total of $180 \mathrm{ml}$. on each of the three days. The order of administration was reversed in $\sigma$ of the 13 subjects; thus aspirin plus alcohol was taken on the $6 \mathrm{th}, 7 \mathrm{th}$, and 8 th days, and aspirin alone on the $14 \mathrm{th}, 15 \mathrm{th}$, and 16th days.

* Honorary Assistant Physician.

+ Senior Research Fellow.

A. W. Morrow Department of Gastrcenterology, Royal Prince Alfred Hospital, Sydney, Australia.
The effect of alcohol ingestion alone was studied in seven other male subjects. Faeces were collected for 12 consecutive days and alcohol was taken as described above on the 6th, 7 th, and 8th days.

Specimens were counted in a high geometry counter having a liquid phosphor (ARMAC., Packard Instrument Co., Illinois). For the purpose of statistical comparison the first part of the study was divided into three six-day periods-days 1 to 6 , days 7 to 12 , and daýs 15 to 20 . In the experiments using alcohol alone days 1 to 6 served as control and days 7 to 12 as the experimental period. These divisions were made to allow for the usual delayed appearance of blood in the faeces from upper gastrointestinal bleeding. The mean daily faecal blood loss in each of these periods was calculated by dividing the total blood loss in each period by six. The difference in faecal blood loss during control and experimental periods was evaluated by the $t$ test (Snedecor and Cochran, 1967).

\section{Results}

In each of the 13 subjects the mean daily faecal blood loss was increased after aspirin ingestion (Table I). Mean daily faecal blood loss for all 13 subjects during the control period was $0.4 \pm 0.04 \mathrm{ml}$., during the aspirin period it was $3.2 \pm 0.6 \mathrm{ml}$. In response to alcohol plus aspirin the faecal blood loss in 11 of the 13 subjects was greater than that during the aspirin period. Mean daily faecal blood loss in response to aspirin plus alcohol $(5.3 \pm 0.5 \mathrm{ml}$.) was significantly greater than that in response to aspirin alone $(\mathrm{P}<0.01)$.

TABLE I.-Effect of Aspirin and Aspirin Plus Alcohol on Mean Daily Faecal Blood Loss (ml./Day)

\begin{tabular}{c|c|c|c}
\hline Subject & Control Period & Aspirin Period & Alcohol + Aspirin \\
\hline 1 & 0.4 & 1.4 & 4.5 \\
2 & 0.2 & 0.7 & 2.7 \\
3 & 0.6 & 4.9 & 5.0 \\
4 & 0.4 & 3.4 & 7.8 \\
5 & 0.3 & 2.3 & 3.7 \\
6 & 0.3 & 5.7 & 7.2 \\
7 & 0.4 & 1.3 & 7.5 \\
8 & 0.2 & 2.7 & 2.9 \\
9 & 0.2 & 2.8 & 6.8 \\
19 & 0.5 & 3.8 & 7.0 \\
11 & 0.5 & 8.5 & 3.3 \\
12 & 0.2 & 1.3 & 5.3 \\
\hline 13 & 0.4 & 3.2 & 0.5 \\
\hline Mean & 0.4 & 0.6 & \\
\hline S.E.M. & 0.04 & &
\end{tabular}

In the seven other subjects to whom alcohol alone was given there was no significant increase in the mean daily faecal blood loss with alcohol ingestion (Table II).

TABLE II.-Effect of Alcohol on Mean Daily Faecal Blood Loss (ml./Day)

\begin{tabular}{c|c|c}
\hline Subject & Control Period & Alcohol Period \\
\hline 14 & 0.2 & 0.3 \\
15 & 0.2 & 0.3 \\
16 & 1.8 & 0.6 \\
17 & 0.2 & 0.2 \\
18 & 0.4 & 0.6 \\
19 & 0.2 & 1.9 \\
20 & 0.2 & 0.8 \\
\hline Mean & 0.5 & 0.7 \\
\hline
\end{tabular}




\section{Discussion}

Although there has been extensive investigation of the various effects of alcohol in man, no studies of the effect of alcohol on gastrointestinal blood loss have been published.

In the present investigation gastrointestinal blood loss after ingestion of alcohol did not differ from that during the control period in the seven subjects studied (Table II). Leonards (personal communication, 1968) has also been unable to produce gastrointestinal bleeding in normal subjects after ingestion of alcohol. It is possible, however, that the concentration of alcohol in contact with gastric mucosa may be a critical factor.

In the present study alcohol when given alone did not cause gastrointestinal bleeding, but in the same dosage it increased aspirin-induced gastrointestinal blood loss (Table I). Gastrointestinal blood loss induced by unbuffered aspirin is variable from individual to individual, but Croft and Wood (1967) found that the bleeding response of each individual subject was reproducible. They studied gastrointestinal bleeding due to aspirin ingestion in 21 subjects on two occasion 3 and found the mean daily faecal blood loss to be 6.3 and $6.5 \mathrm{ml}$. Thus in the present study the statistically significant increase in gastrointestinal blood loss associated with ingestion of alcohol plus aspirin was almost certainly due to the addition of alcohol and not to variation in bleeding in response to aspirin alone.
The mechanism of action of alcohol on gastrointestinal blood loss induced by aspirin was not studied in the present experiments. Davenport (1965) found that in dogs with a Heidenhain pouch aspirin caused bleeding from the pouch only if the $\mathrm{pH}$ of the contents was acid. As alcohol is a known stimulant of gastric acid secretion it is possible that the increased bleeding with alcohol plus aspirin was a result of lowered $\mathrm{pH}$ of the gastric contents during the period aspirin was in the stomach.

We are grateful to Miss Angela Birchall and Miss Irena Lopata for technical assistance, to the subjects for their co-operation, and to Mr. David Paix, physicist, Prince Henry Hospital, for advice and for the use of the ARMAC counter. One of us (K. G.) was supported by an Abbott Fellowship of the Royal Australasian College of Physicians, and another (A. R. C.) by the National Health and Medical Research Council, Australia. This work was supported in part by the Bushell Trust.

\section{REFERENCES}

Croft, D. N., and Wood, P. H. N. (1967). Brit. med. 7., 1, 137.

Davenport, H. W. (1965). Gastroenterology, 49, 189.

Goulston, K., and Skyring, A. (1964). Gut, 5, 463.

Smith, M J H., and Smith, P. K. (1966). The Salicylates. New York. Snedecor, G. W., and Cochran, W. G. (1967). Statistical Methods, 6th ed. Ames, Iowa.

\title{
Serum Fibrin Degradation Products throughout Normal Pregnancy
}

\author{
D. G. WOODFIELD,* M.B., M.R.C.P.ED. ; S. K. COLE, $†$ M.B., M.R.C.o.G. ; A. G. E. ALLAN, $\ddagger$ F.I.M.L.T. \\ J. D. CASH,§ M.B., PH.D., M.R.C.P.ED.
}

Brit. med.7., 1968, 4, 665-668

\begin{abstract}
Summary : Fibrin/fibrinogen degradation products in the serum of 169 healthy pregnant and 43 healthy non-pregnant subjects of a similar age group showed a significant increase throughout pregnancy, the highest levels being obtained in the third trimester. There was no correlation with plasma fibrinogen levels or euglobulin lysis times. Though these changes may be due to other alterations in pregnancy not primarily associated with fibrinolysis, it is possible that actual fibrinolysis is not decreased in late pregnancy, as has been previously asssumed.
\end{abstract}

\section{Introduction}

The human fibrinolytic enzyme system in normal pregnancy has been studied extensively in the past decade. Most investigators are now agreed that those methods considered to assess the level of circulating plasminogen activator will readily show a marked diminution of this enzyme in the latter part of the gestational period (Biezenski and Moore, 1958; Gillman et al., 1959 ; Biezenski, 1960 ; Shaper et al., 1965; Nilsson and Kullander, 1967). Furthermore, other groups have confirmed the earlier findings of Brakman and Astrup (1963) that the plasma urokinase inhibitor activity is increased in the third trimester of pregnancy (Kawano et al., 1968; Shaper et al.,

* Registrar.

+ Registrar, Simpson Memorial Maternity Pavilion, Royal Infirmary, Edinburgh 3.

¥ Senior Technician.

Deputy Director.

outh-east Scotland Regional Blood Transfusion Centre, Royal Infirmary, Edinburgh 3.
1968). Moreover, Brakman (1966) has presented evidence which indicates that the binding of the plasminogen activatorcomplex, envisaged by Olesen (1961), is also increased in the third trimester.

Plasminogen activators are believed to play a key part in promoting in-vivo fibrinolysis (Fearnley, 1953 ; Sherry et al., 1959), whereas fibrinolytic inhibitors retard this process (Naeye, 1961 ; Nilsson et al., 1961 ; Turpie et al., 1967). In the light of the evidence currently available it would therefore be reasonable to assume that the actual lysis of fibrin in the latter months of pregnancy is in fact depressed. Proof of this is lacking, however, for the methods used to accumulate this evidence have assessed only the absolute circulating levels of the various fibrinolytic components and have provided relatively little information on their in-vivo dynamic interactions.

The recent introduction of the tanned red-cell haemagglutination inhibition immunoassay for the quantitative assessment of circulating fibrin/fibrinogen degradation products (F.D.P.) (Murakami, 1965; Merskey et al., 1966) and its subsequent development to enable the detection of F.D.P. levels in over $95 \%$ of normal sera (Das et al., 1967) now allows a further investigation of the fibrinolytic enzyme system in pregnancy, for it can be assumed that the level of serum F.D.P. represents a measure of active in-vivo fibrinolysis.

The following communication describes the results of an investigation designed to ascertain the level of serum F.D.P. in 169 normal pregnant women in order to test the hypothesis discussed above and to provide normal values as guide lines for the diagnosis of those haemorrhagic emergencies which occasionally complicate pregnancy. 